\title{
Local delivery of tetracycline from $y$-aminobutiric acid-silica networks thin films for preventing microbial colonization
}

https://doi.org/10.33263/BTEB114.002009

\author{
Alina Maria Holban ${ }^{1,2}$, Ecaterina Andronescu ${ }^{2}$, Valentina Grumezescu ${ }^{2,3}$, Gabriel Socol ${ }^{3}$, \\ Alexandru Mihai Grumezescu ${ }^{1, *}$, Veronica Lazăr ${ }^{2}$, Mariana Carmen Chifiriuc ${ }^{2}$ \\ 1 Department of Science and Engineering of Oxide Materials and Nanomaterials, Faculty of Applied Chemistry and Materials \\ Science, University Politehnica of Bucharest, 1-7 Polizu Street, 011061 Bucharest, Romania \\ 2 Microbiology Immunology Department, Faculty of Biology, University of Bucharest, 1-3 Portocalilor Lane, Sector 5, 77206 \\ Bucharest, Romania \\ 3 Lasers Department, National Institute for Lasers, Plasma \& Radiation Physics, P.O. Box MG-36, Magurele, Bucharest, Romania \\ *corresponding author e-mail address: grumezescu@yahoo.com
}

\section{ABSTRACT}

The purpose of this study was to characterize and evaluate the citotoxicity and antimicrobial activity of $\gamma^{-}$ aminobutiric acid-silica/tetracycline network thin films prepared by Matrix Assisted Pulsed Laser Evaporation (MAPLE). Thin films were characterized by Infrared Microscopy (IRM), X-ray Diffraction (XRD), Transmision Electron Microscopy (TEM) and Scanning Electron Microscopy (SEM). TEM images showed an average size of gammaaminobutiric acid-silica network $\left(\gamma-\mathrm{AA} / \mathrm{SiO}_{2}\right)$ lower than $10 \mathrm{~nm}$. Unlike the dropcast samples, IRM recorded on MAPLE films revealed a good distribution of monitored functional groups on the entire scanned surface. Besides a good homogeneity of the coatings, SEM analysis revealed aggregates typical for MAPLE deposition, randomly distributed on the surface with an average diameter of $\sim 300 \mathrm{~nm}$. The biological evaluation of MAPLE nanobiocoated surfaces evidenced a good biocompatibility and an enhanced antibiofilm effect against both Gram positive (Staphylococcus aureus) and Gram negative (Escherichia coli) tested strains. Our results recommend the use of $\gamma$-aminobutiric acid-silica networks as matrixes for controlled local delivery of antibiotics, with practical applications in developing improved medical surfaces for the prevention or reduction of surface-associated microbial infections.

Keywords: antimicrobial surfaces, MAPLE, S. aureus, E. coli, $\gamma$-aminobutiric acid-silica networks.

\section{INTRODUCTION}

Hospital surfaces contamination has a major impact in the transmission of healthcareassociated pathogens, especially multidrug resistant bacteria. Even though major interventions have been made in order to improve cleaning and disinfestations in healthcare facilities, hospitalacquired infections remain a major cause of patient morbidity and mortality worldwide (Boyce, 2007; Klevens et al., 2007). In the US only, it is estimated a number of 1.7 million healthcareassociated infections, which result in approximately 99000 deaths per year (Klevens et al., 2007). Studies demonstrated that main sources of pathogens causing hospital-acquired infections are: i) the patients' endogenous flora (40-60\%); ii) cross-infection via the hands of personnel and contaminated medical objects (20-40\%); iii) antibiotic-driven modifications in patients' microbiota (20-25\%); and iv) other, including contamination of the healthcare unit environment (20\%) (Weber et al., 2013). Studies proved that the contaminated surfaces in hospitals play an important role in the transmission of methicillinresistant Staphylococcus aureus (MRSA), vancomycin-resistant Enterococcus spp. (VRE), Clostridium difficile, Acinetobacter spp., and norovirus (Weber et al., 2013).

The key aspects demonstrating the importance of surface contamination in the transmission of hospital - acquired infections are 
explained by the following: i) surfaces in a rooms of colonized or infected patients are frequently contaminated with the pathogen; ii) the pathogen is able to survive long periods of time on hospital room surfaces and medical equipment; iii) the contact of healthcare personnel with infected medical surfaces or equipment leads to contamination of hands or gloves; iv) person-toperson transmission and shared medical equipment is the major cause of clonal outbreaks of pathogens contaminating the medical surfaces of colonized or infected patients (Weber et al., 2013).

Furthermore, another risk condition for acquiring healthcare-associated infections is represented by patients with implanted medical and prosthetic devices. Due to their wide spread within the body and skin, Staphylococci and Escherichia coli account for the majority of deviceassociated infections (von Eiff et al., 2005). Their ability to adhere to materials and to promote biofilms formation is the most important feature of their pathogenicity. Since biofilms are highly resistant structures to most antimicrobials and host defense system (Lazar, 2011), the removal of infected implanted devices is often inevitable.

To reduce the incidence of device-associated infections, specific guidelines including both technological and non technological strategies for prevention have been established (Cotar et al., 2013; Grumezescu and Chifiriuc, 2014; Holban et al., 2014). The surface modification of the implantable device may lead to a change in its specific and nonspecific interactions with microorganisms, and thus to the reduction of microbial adherence and biofilms formation. A new and promising strategy involves the impregnation of the devices with various natural and synthetic antimicrobial substances in order to prevent colonization (von Eiff et al., 2005).

Matrix Assisted Pulsed Laser Evaporation (MAPLE) has proved a great impact on surface coating, allowing the deposition of thin, uniform films, made of different nanostructured materials, polymers and active compounds (Grumezescu et al., 2014c; Grumezescu et al., 2014d).

$\gamma$-aminobutyric acid $(\gamma-A A)$ is a non-protein amino acid widespread in the environment. Being a bifunctional molecule, $\gamma$-AA can be used in nanotechnology to obtain nanostructured networks with low diameter pores (Grumezescu et al., 2014a). Also, many bacteria, such as Pseudomonas sp, lactic bacteria and Escherichia coli, can synthesize and release $\gamma-A A$, suggesting that this compound may act as a communication molecule between different, or between bacteria and their host (Dagorn et al., 2013). Therefore, utilizing $\gamma$-AA for obtaining nanostructurated materials with antimicrobial effect we can increase not only the quality of the material, by controlling the pore size, but also the efficiency of the material on bacteria, which are familiar with such compounds.

Silica networks as drug delivery system, were involved in the development of new formulations that control rate and period of drug delivery (Heikkila et al., 2007; Li et al., 2014; Peng et al., 2013). Few studies reported the potential of silica networks to improve the antimicrobial effect of currently used antibiotics (Balaure et al., 2013; Grumezescu et al., 2013; Guzun et al., 2014; Voicu et al., 2013) and fewer studies report the laser processing of silica network in order to create antimicrobial surfaces (Mihaiescu et al., 2013).

The purpose of this study was the fabrication, characterization and bioevaluation of $\gamma$-aminobutiric acid-silica network thin film prepared by MAPLE as a matrix for controlled local delivery of the antibiotic tetracycline, with practical applications in developing improved medical surfaces for the prevention or reduction of surfaceassociated infections.

\section{MATERIALS AND METHODS}

\section{Materials and methods}

The $\gamma$-aminobutyric acid (>99\%), sodium metasilicate, sulfuric acid (ACS reagent 95-98\%), were purchased from Sigma-Aldrich, and were used without any further purification. 


\section{Preparation of $\gamma$-aminobutiric acid-silica network}

The synthesis of $\gamma$-aminobutiric acid-silica (gamma-AA/SiO 2 ) network was performed using our previously reported protocol (Grumezescu et al., 2014a).

MAPLE target preparation and deposition of $\boldsymbol{\gamma}$ aminobutiric acid-silica networks@tetracycline thin films

$400 \mathrm{mg} \gamma$-aminobutiric acid-silica networks and $100 \mathrm{mg}$ tetracycline were dispersed in $30 \mathrm{~mL}$ DMSO then the solution was poured into a precooled target holder and subsequently immersed in liquid nitrogen for $30 \mathrm{~min}$. MAPLE depositions were carried out in a stainless steel chamber using a $\mathrm{KrF}^{*}$ laser source $\left(\lambda=248 \mathrm{~nm}\right.$ and $\left.\tau_{\mathrm{FWHM}}=25 \mathrm{~ns}\right)$, model COMPexPro 205 (Lambda PhysicsCoherent), that operated at a repetition rate of 15 $\mathrm{Hz}$. A laser beam homogenizer was used to improve the energy distribution of the laser spot. The laser fluence was set at $300-500 \mathrm{~mJ} / \mathrm{cm}^{2}$ range whereas the laser spot area was set to $36 \mathrm{~mm}^{2}$. During the laser irradiation, the frozen target was rotated at a rate of $0.4 \mathrm{~Hz}$ to avoid the target heating and drilling. All depositions were conducted at room temperature into a background pressure of $1 \mathrm{~Pa}$. The films were grown at a target-substrate separation distance of $5 \mathrm{~cm}$ by applying 140,000 subsequent laser pulses. During the deposition process, the target was kept at a temperature of $\sim 173 \mathrm{~K}$ by active liquid nitrogen cooling. The coatings were deposited onto double side polished (100) silicon and glass substrates for physico-chemical analyses and biological assays, respectively. Prior to introduction inside the deposition chamber, the substrates were successively cleaned into an ultrasonic bath with acetone, ethanol and deionized water for $15 \mathrm{~min}$. During the deposition, the substrates were continuously rotated. Thus, the $\gamma$-aminobutiric acid-silica networks/tetracycline was uniformly spread over the surface of the substrates. For data comparison, a control set of films were prepared by pouring of few drops onto the surface of double side polished (100) silicon.

\section{Characterization}

XRD

X-ray diffraction analysis was performed on a Shimadzu XRD 6000 diffractometer at room temperature. In all the cases, $\mathrm{K}$ radiation from a $\mathrm{Cu}$ $\mathrm{X}$-ray tube (run at $15 \mathrm{~mA}$ and $30 \mathrm{kV}$ ) was used. The samples were scanned in the $(2 \theta)$ Bragg-Brentano geometry in the $10-40^{\circ}$ range.

TEM

The transmission electron microscopy (TEM) images were obtained on finely powdered samples using a Tecnai ${ }^{\mathrm{TM}}$ G2 F30 S-TWIN high resolution transmission electron microscope from $\mathrm{FEI}$ Company (OR, USA) equipped with SAED. The microscope operated in transmission mode at 300 $k V$ with TEM point resolution of $2 \AA$ and line resolution of $1 \AA$. The prepared powder was dispersed into pure ethanol and ultrasonicated for $15 \mathrm{~min}$. After that, diluted sample was poured onto a holey carbon-coated copper grid and left to dry before TEM analysis.

\section{IRM}

IR mapping were recorded on a Nicolet iN10 MX FT-IR Microscope with MCT liquid nitrogen cooled detector in the measurement range 4000$700 \mathrm{~cm}^{-1}$. Spectral collection was made in reflection mode at $4 \mathrm{~cm}^{-1}$ resolution. For each spectrum, 32 scans were co-added and converted to absorbance using OmincPicta software (Thermo Scientific). Approximately 250 spectra were analyzed for each sample. One absorption peak known as being characteristics for the prepared material was selected as spectral markers.

\section{SEM}

SEM analysis was performed on a FEI electron microscope, using secondary electron beams with energies of $30 \mathrm{keV}$, on samples covered with a thin gold layer.

\section{In vitro biocompatibility of $\boldsymbol{y}$-aminobutiric acid-silica network thin films}

The biocompatibility of $\gamma$-aminobutiric acidsilica networks-based thin films with endothelial cells was asses by MTT Assay. Briefly, endothelial cells (EAhy926 cell line, ATCC) were grown on $y^{-}$ aminobutiric acid-silica networks-based thin films substrates for 24 hours and viability measurements were done using a CellTiter96 Non-Radioactive Cell Proliferation MTT Assay kit (Promega, 
Madison, USA), following the manufacturer's instructions. Cells proliferation was assess through spectrophotometry measurements at $570 \mathrm{~nm}$ using a Mithras LB 940 apparatus (Berthold Technology, Germany). For revealing cell morphology and viability, fluorescent microscopy was assessed using a RED CMTPX fluorophore (Life Technologies, Invitrogen, USA), a cell tracker for long-term tracing of living cells. The RED CMTPX dye was added in the culture medium at a final concentration of $5 \mu \mathrm{M}$ and incubated 30 minutes for allowing the dye to penetrate the cells. Furthermore, the cells were washed with PBS and visualized by fluorescent microscopy. The photomicrographs were taken with a digital camera driven by the Axio-Vision 4.6 (Carl Zeiss, Germany) software.

\section{Biofilm development assay}

The strains used in this study, S. aureus ATCC 25923 and E. coli ATCC 25922 were obtained from the American Type Culture Collection (ATCC, US). Strains from glycerol stocks were streaked on nutritive agar plates and colonies were allowed to develop for $24 \mathrm{~h}$ at $37^{\circ} \mathrm{C}$. Fresh colonies were used to obtain a bacterial suspension of a $0,5 \mathrm{Mc}$ Farland (corresponding to $\sim 10^{8} \mathrm{CFU} / \mathrm{mL}$ ) optical density in phosphate buffered saline (PBS). For assessing monospecific biofilms formation, $2 \mathrm{~mL}$ of nutritive broth were disposed in each well of a 6 wells plate, containing test ( $\gamma$-aminobutiric acidsilica networks thin films coatings) and control (bare glass substrates). Bacterial inoculum consisted of a volume of $20 \mu \mathrm{L}$ from the PBS bacterial suspension, added in the $2 \mathrm{~mL}$ of the nutritive broth. After a period of $24 \mathrm{~h}$ incubation at $37^{\circ} \mathrm{C}$, the materials containing attached bacteria, were washed with PBS and transferred in a fresh well, containing $2 \mathrm{~mL}$ sterile nutritive broth. Biofilm formation on the materials was assessed after 24, 48 and $72 \mathrm{~h}$ of incubation by the viable cell count (VCC) method. For this, after each time point, biofilm embedded bacteria cells were detached by vigorous vortexing for 30 seconds. PBS suspensions containing detached bacteria cells were subjected to serial dilutions and each dilution was seeded on nutritive agar. Experiments were performed in triplicate and repeated on at least three separate occasions.

\section{RESULTS AND DISCUSSIONS}

Recently, we reported on the synthesis and characterization of $\gamma-\mathrm{AA} / \mathrm{SiO}_{2}$ nanoparticles with a diameter that not exceed $10 \mathrm{~nm}$ and with the pores diameter $\sim 4.76 \mathrm{~nm}$ (Grumezescu et al., 2014a). Figure 1 presents the XRD pattern of $\gamma$ $\mathrm{AA} / \mathrm{SiO}_{2}$. The pattern reveals a broad peak in the $15-35^{\circ}$ range that can be assigned to silica with low degree of cristalinity.

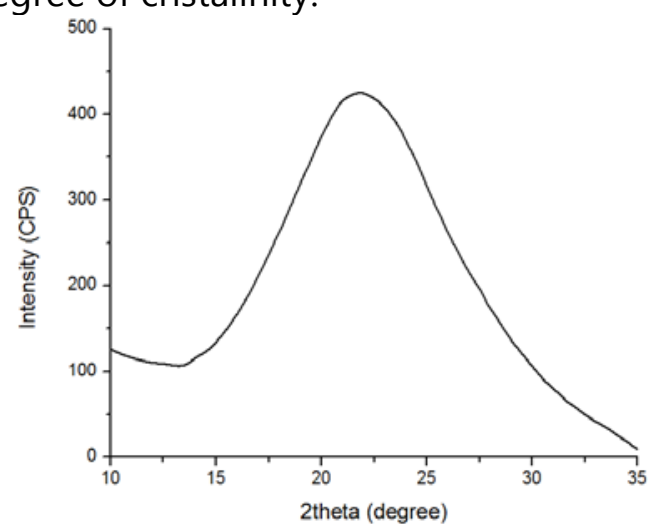

Figure 1. XRD pattern of gamma-AA/SiO .

TEM images of the prepared gamma$\mathrm{AA} / \mathrm{SiO}_{2}$ showed in figure 2 confirm the XRD results, the size of nanoparticles being under 10 $\mathrm{nm}$. All these results are in good agreement with our previous published paper (Grumezescu et al., 2014a).

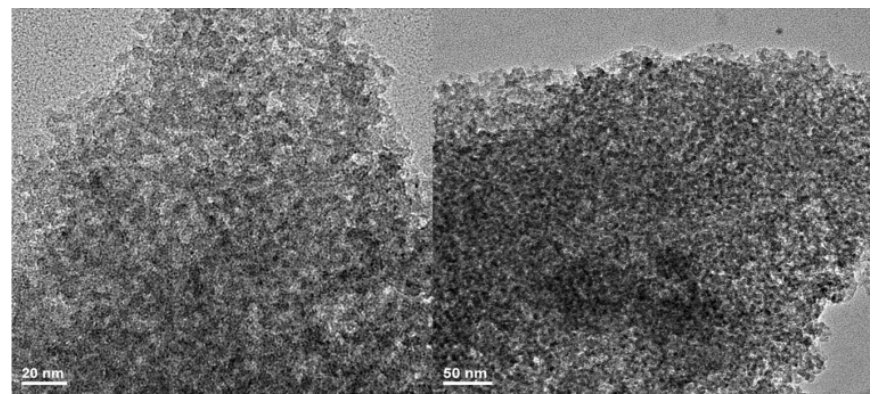

Figure 2. TEM images of gamma-AA/SiO ${ }_{2}$.

Infrared Microscopy was used to evaluate the integrity of functional groups of the prepared thin film by comparison with dropcast samples. For further investigations, the laser fluence at 400 $\mathrm{mJ} / \mathrm{cm}^{2}$ was selected as a compromise between the deposition rate and the stoichiometric transfer (Grumezescu et al., 2014b). Figure 3 and 4 present 
IR maps of dropcast and MAPLE thin films. The monitored band was characteristic to tetracycline $\left(1616 \mathrm{~cm}^{-1}\right)$. According to these figures it can be concluded that the thin films were successfully deposed without organic molecules degradation.

In the FTIR spectra we observed that the absorption bands are characteristics to silica ( $\mathrm{Si}-\mathrm{O}$ , $\left.1057 \mathrm{~cm}^{-1}\right)$ and to tetracycline $\left(C=0,1616 \mathrm{~cm}^{-1}\right)$. The broad peak between $3200-2900 \mathrm{~cm}^{-1}$ typical to the vibration of $\mathrm{OH}$ group is also visible for dropcast and MAPLE samples.
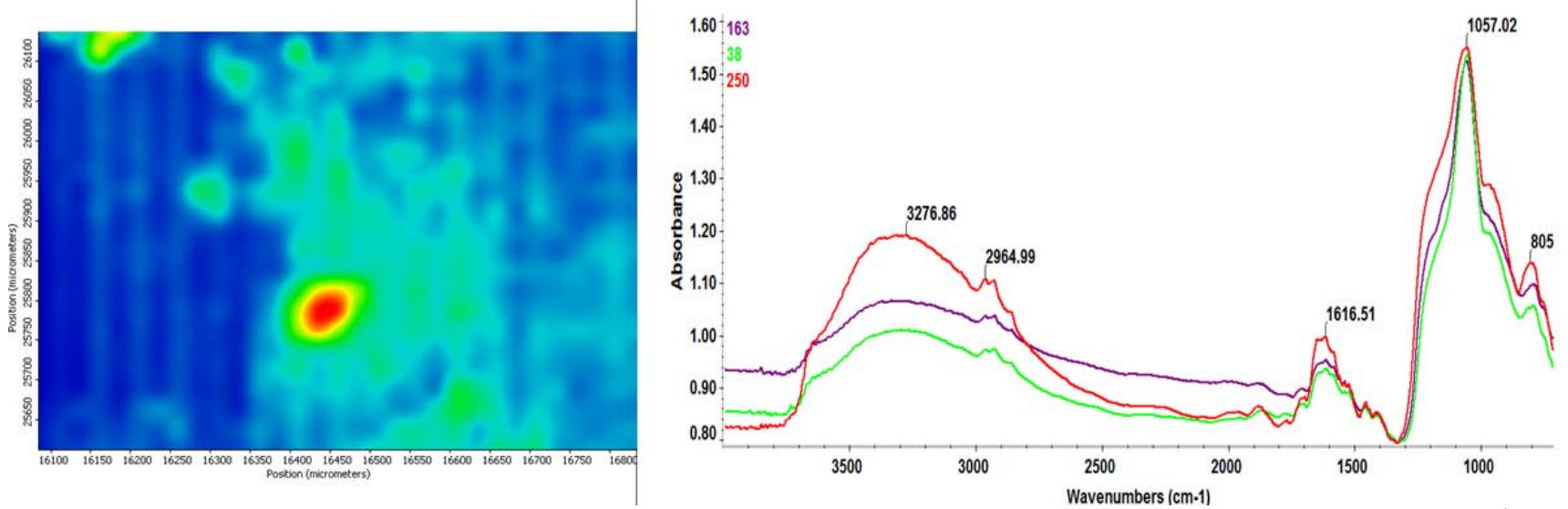

Figure 3. Second IR derivate mapping and IR spectra of gamma-AA/SiO $/$ TET dropcast sample: intensity distribution of $1616 \mathrm{~cm}^{-1}$.
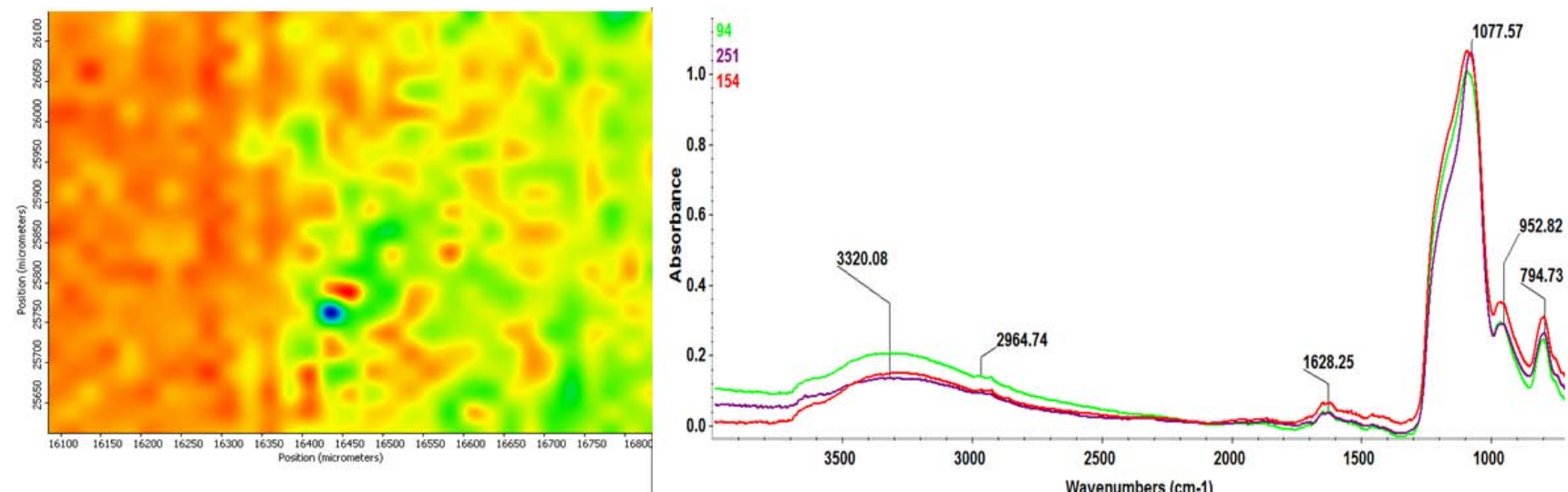

Figure 4. Second IR derivate mapping and IR spectra of gamma-AA/SiO ${ }_{2} / \mathrm{TET}$ thin film $\left(\mathrm{F}=400 \mathrm{~mJ} / \mathrm{cm}^{2}\right)$ : intensity distribution of 1616 $\mathrm{cm}^{-1}$.

Figure 5 shows de SEM images for the gamma$\mathrm{AA} / \mathrm{SiO}_{2} / \mathrm{TET}$ thin film. At low magnification, the thin film display a homogeneous morphology with small rounded aggregates with the size within the range $200-300 \mathrm{~nm}$ uniformly distributed on the

surface. These results are in good agreements our previous results (Grumezescu et al., 2014b). The MAPLE processing of silica precursors/networks creates micro- and nano-particulates (Grumezescu et al., 2014b).

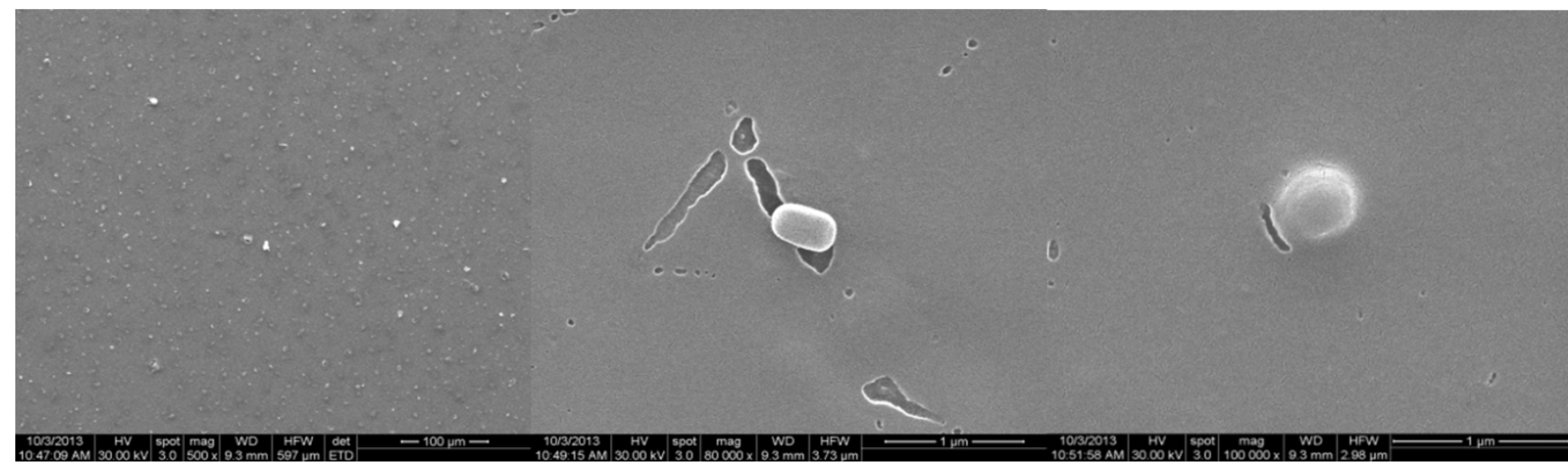

Figure 5. Typical SEM images of gamma-AA-SiO ${ }_{2} / \mathrm{TET}$ coatings. 
Fluorescence

microscopy

assay demonstrated that $\gamma$-AA-SiO $2 /$ TET coatings does not modify the morphology and viability rate of endothelial cells grown on these modified surfaces for $24 \mathrm{~h}$ (figure 6).
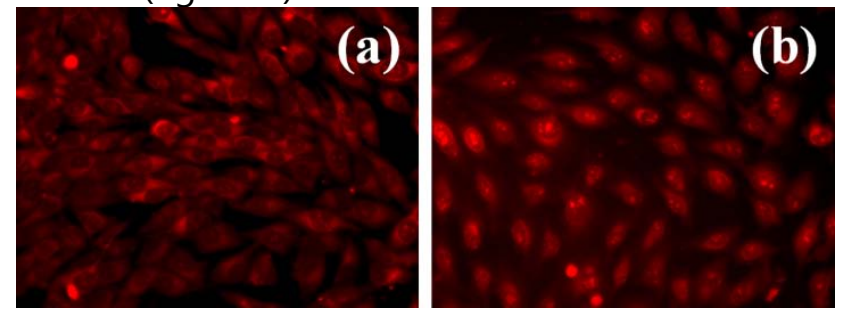

Figure 6. Florescence microscopy images of human endothelia cells developed on gamma-AA-SiO ${ }_{2} /$ TET coatings (a) and control (b) surfaces for $24 \mathrm{~h}$.

Furhtermore, MTT assay evidenced that endothelial cells grown on $\gamma-\mathrm{AA} / \mathrm{SiO}_{2} / \mathrm{TET}$ develop a normal proliferation rate and show an adequate metabolism rate, similar with the cells grown on the controls (bare glass substrates) (figure 7). These results demonstrate a good biocompatibility of this material with respect to human endothelial cells.

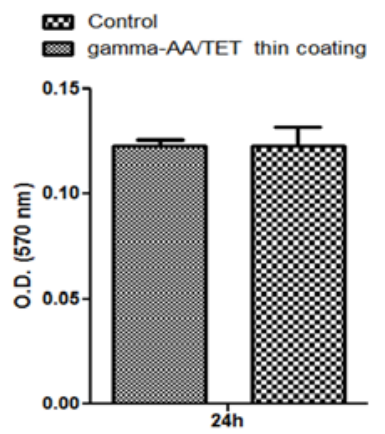

Figure 7. Graphic representation of quantitative evaluation of the human endothelial cells proliferation rates in the presences of gamma-AA-SiO ${ }_{2} /$ TET coatings and control surfaces.

Even though the MAPLE fabricated thin coatings have no citotoxic effects on cultured human cells, they proved a great effect on bacterial cell. The enhanced antimicrobial behavior may be explained by the fact that $-\mathrm{AA}-\mathrm{SiO}_{2} / \mathrm{TET}$ thin coatings ensure the controlled release of the antibiotic, enhancing its efficiency. Both S. aureus and $E$. coli biofilms development was impaired on the coated surfaces, the results revealing that the biofilm inhibitory effect is maintained along the bacterial incubation time. The most significant biofilm reduction was observed at the initial stages of biofilm development, after $24 \mathrm{~h}$ of incubation.
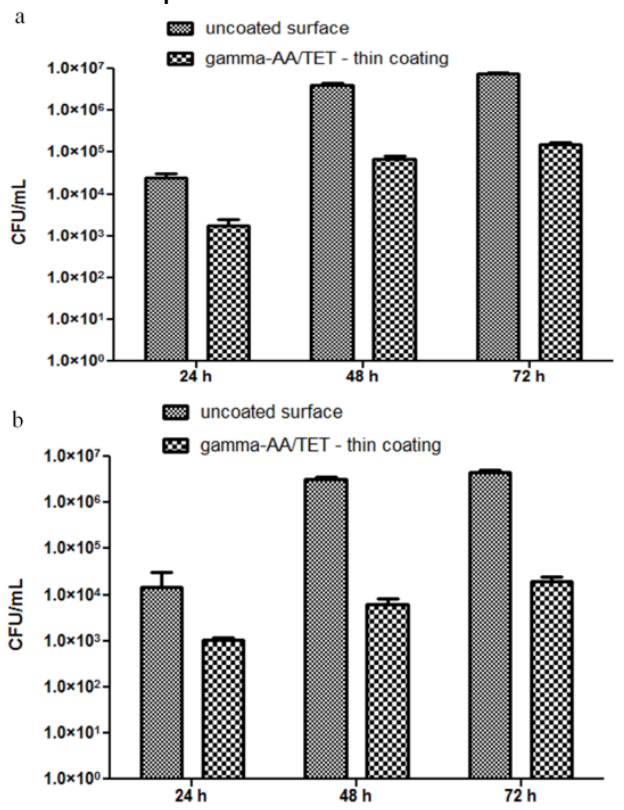

Figure 8. Graphic representation of $S$. aureus (a) and E. coli (b) biofilm development on the control and nano-coated surfaces.

This result may be explained by the fact that the release of the antibiotic from the $\gamma-\mathrm{AA}-\mathrm{SiO}_{2}$ matrix is more pronounced in the early hours of biofilm development, or that the material inhibits bacterial initial attachments. We also observed for both $S$. aureus and E. coli a slight decrease in the antibiofilm efficiency of the fabricated coating during time, but the antimicrobial effect is still high enough to reveal a significant difference in the biofilms developed on the control and nanobiocoated surfaces (figure 8).

\section{CONCLUSIONS}

Thin films based on silica networks/tetracycline were successfully transferred by MAPLE technique. SEM analysis reveals a good homogeneity of the sample with rounded particulates of 200-300 nm randomly distributed on the surface. The MAPLE thin films significantly improved the resistance to microbial colonization, inhibiting the biofilm formation on both Gram positive (S. aureus) and Gram negative (E. coli) tested strains. These results correlated with their high biocompatibility highlight the possibility of using the $\gamma$-aminobutiric acid-silica network films 
for the controlled local delivery of the therapeutic agents in low active doses that it may diminish the occurrence of infection and rejection for implanted prosthetic devices.

\section{REFERENCES}

Balaure, P.C., Andronescu, E., Grumezescu, A.M., Ficai, A., Huang, K.S., Yang, C.H., Chifiriuc, C.M., and Lin, Y.S. (2013). Fabrication, characterization and in vitro profile based interaction with eukaryotic and prokaryotic cells of alginate-chitosan-silica biocomposite. Int J Pharm 441, 555-561.

Boyce, J.M. (2007). Environmental contamination makes an important contribution to hospital infection. J Hosp Infect 65, 50-54.

Cotar, A.I., Grumezescu, A.M., Huang, K.-S., and Voicu, G. (2013). Magnetite nanoparticles influence the efficacy of antibiotics against biofilm embedded Staphylococcus aureus cells. Biointerface Research in Applied Chemistry.

Dagorn, A., Chapalain, A., Mijouin, L., Hillion, M., Duclairoir-Poc, C., Chevalier, S., Taupin, L., Orange, N., and Feuilloley, M.G.J. (2013). Effect of GABA, a Bacterial Metabolite, on Pseudomonas fluorescens Surface Properties and Cytotoxicity. Int J Mol Sci 14, 12186-12204.

Grumezescu, A.M., Andronescu, E., Ficai, A., Grumezescu, V., Bleotu, C., Saviuc, C., Mihaiescu, D.E., and Chifiriuc, C.M. (2013). Biocompatible Magnetic Hollow Silica Microspheres for Drug Delivery. Curr Org Chem 17, 1029-1033.

Grumezescu, A.M., and Chifiriuc, C.M. (2014). Prevention of microbial biofilms - the contribution of micro and nanostructured materials. Current medicinal chemistry 21, 3311.

Grumezescu, A.M., Ghitulica, C.D., Voicu, G., Huang, K.S., Yang, C.H., Ficai, A., Vasile, B.S., Grumezescu, V., Bleotu, C., and Chifiriuc, M.C. (2014a). New silica nanostructure for the improved delivery of topical antibiotics used in the treatment of staphylococcal cutaneous infections. Int J Pharmaceut 463, 170-176.

Grumezescu, V., Ficai, A., Holban, A.M., Socol, G., Grumezescu, A.M., Ficai, D., Grosu, E., Mihailescu, I., Bleotu, C., and Chifiriuc, M.C. (2014b). MAPLE deposition of silicon based polymer coatings on PVC with anti-adherent properties. E-MRS 2013 FALL MEETING, Symposium I: Multifunctional Bionanomaterials, September 16-19, Warsaw, Poland.

Grumezescu, V., Holban, A.M., Grumezescu, A.M., Socol, G., Ficai, A., Vasile, B.S., Trusca, R., Bleotu, C., Lazar, V., Chifiriuc, C.M., et al. (2014c). Usnic acid-loaded biocompatible magnetic PLGA-PVA microsphere thin films fabricated by MAPLE with increased resistance to staphylococcal colonization. Biofabrication 6, 035002.

Grumezescu, V., Socol, G., Grumezescu, A.M., Holban, A.M., Ficai, A., Trusca, R., Bleotu, C., Balaure, P.C., Cristescu, R., and Chifiriuc, M.C. (2014d). Functionalized antibiofilm thin coatings based on PLA-PVA microspheres loaded with usnic acid natural compounds fabricated by MAPLE. Appl Surf Sci 302, 262-267.

Guzun, A.S., Stroescu, M., Jinga, S.I., Voicu, G., Grumezescu, A.M., and Holban, A.M. (2014). Plackett-Burman experimental design for bacterial cellulose-silica composites synthesis. Materials Science and Engineering: C 42, 280-288.

Heikkila, T., Salonen, J., Tuura, J., Hamdy, M.S., Mul, G., Kumar, N., Salmi, T., Murzin, D.Y., Laitinen, L., Kaukonen, A.M., et al. (2007). Mesoporous silica material TUD-1 as a drug delivery system. Int J Pharmaceut 331, 133-138.

Holban, A.M., Grumezescu, V., Grumezescu, A.M., Vasile, B.Ş., Truşcă, R., Cristescu, R., Socol, G., and Iordache, F. (2014). Antimicrobial nanospheres thin coatings prepared by advanced pulsed laser technique. Beilstein Journal of Nanotechnology 5, 872-880.

Klevens, R.M., Edwards, J.R., Richards, C.L., Jr., Horan, T.C., Gaynes, R.P., Pollock, D.A., and Cardo, D.M. (2007). Estimating health care-associated infections and deaths in U.S. hospitals, 2002. Public health reports (Washington, DC : 1974) 122, 160-166.

Lazar, V. (2011). Quorum sensing in biofilms - How to destroy the bacterial citadels or their cohesion/power? Anaerobe 17, 280-285.

Li, J., Xu, L., Liu, H.Z., Wang, Y., Wang, Q.F., Chen, H.T., Pan, W.S., and Li, S.M. (2014). Biomimetic synthesized nanoporous silica@poly(ethyleneimine)s xerogel as drug carrier: Characteristics and controlled release effect. Int J Pharmaceut 467, 9-18.

Mihaiescu, D.E., Cristescu, R., Dorcioman, G., Popescu, C.E., Nita, C., Socol, G., Mihailescu, I.N., Grumezescu, A.M., Tamas, D., Enculescu, M., et al. (2013). Functionalized magnetite silica thin films fabricated by MAPLE with antibiofilm properties. Biofabrication 5.

Peng, H.L., Dong, R.C., Wang, S.Q., Zhang, Z., Luo, M., Bai, C.Q., Zhao, Q., Li, J.H., Chen, L.X., and Xiong, H. (2013). A pH-responsive nano-carrier with mesoporous silica nanoparticles cores and 
poly(acrylic acid) shell-layers: Fabrication, characterization and properties for controlled release of salidroside. Int J Pharmaceut 446, 153-159.

Voicu, G., Grumezescu, V., Andronescu, E., Grumezescu, A.M., Ficai, A., Ficai, D., Ghitulica, C.D., Gheorghe, I., and Chifiriuc, M.C. (2013). Caprolactam-silica network, a strong potentiator of the antimicrobial activity of kanamycin against Gram-positive and Gram-negative bacterial strains. Int J Pharmaceut 446, 63-69. von Eiff, C., Jansen, B., Kohnen, W., and Becker, K. (2005). Infections associated with medical devices Pathogenesis, management and prophylaxis. Drugs 65, 179-214.

Weber, D.J., Anderson, D., and Rutala, W.A. (2013). The role of the surface environment in healthcareassociated infections. Curr Opin Infect Dis 26, 338344.

\section{Conflicts of Interest}

The authors declare no conflict of interest.

(C) 2014 by the authors; licensee AMG Transcend, Bucharest, Romania. This article is an open access article distributed under the terms and conditions of the Creative Commons Attribution license (http://creativecommons.org/licenses/by/4.0/). 